\title{
Fracture resistance of CAD/CAM restorative materials in mismatched removable partial denture rests: An in vitro experimental and finite element analysis
}

\author{
Keiichiro UCHIKURA ${ }^{1}$, Natsuko MURAKAMI ${ }^{1}$, Toshiki YAMAZAKI ${ }^{1}$, Huaxin LYU ${ }^{1}$, Kohji NAGATA ${ }^{1}$, Masahiro ONA ${ }^{1}$, \\ Naohiko IWASAKI ${ }^{2}$, Hidekazu TAKAHASHI ${ }^{2}$ and Noriyuki WAKABAYASHI ${ }^{1}$ \\ ${ }^{1}$ Advanced Prosthodontics, Oral Health Sciences, Graduate School of Medical and Dental Sciences, Tokyo Medical and Dental University, 1-5-45 \\ Yushima, Bunkyo-ku, Tokyo 113-8549, Japan \\ ${ }^{2}$ Oral Health Engineering, Graduate School of Medical and Dental Sciences, Tokyo Medical and Dental University, 1-5-45 Yushima, Bunkyo-ku, \\ Tokyo 113-8549, Japan \\ Corresponding author, Natsuko MURAKAMI; E-mail: n.murakami.rpro@tmd.ac.jp
}

To evaluate the fracture resistance of computer-aided design and computer-assisted manufacturing restorations as the abutment of removable partial dentures, experimental blocks, with the rest seat made of feldspar, hybrid resin composite, lithium disilicate glass ceramic, or zirconia, were subjected to loading by a metallic occlusal rest. The rest contacted the rest seat with an accurate fit and two mismatch contact conditions: bottom and sidewall contact. Zirconia exhibited the highest fracture load, and the fracture load of the accurate fit was significantly higher than that of the sidewall contact $(p<0.05)$ and insignificantly higher than that of the bottom contact $(p>0.05)$. A finite element analysis of the sidewall contact revealed a higher tensile stress concentration at the bottom of the rest seat than the other contact conditions. The mismatch between the rest and the restoration reduced fracture resistance, while zirconia as the abutment withstood the average occlusal force of the posterior region.

Keywords: CAD/CAM, Removable partial denture, Rest seat, Fracture load, FEM

\section{INTRODUCTION}

The rapid development of computer-aided design and computer-assisted manufacturing (CAD/CAM) technology has allowed the manufacture of non-metallic, tooth-colored, monolithic restorations, such as ceramics and resin composite materials, with excellent esthetics and fracture strength sufficient to withstand the masticatory force. The abutment tooth of the removable partial denture (RPD) absorbs the masticatory force through the rest, which is part of the framework of the RPD ${ }^{1}$. However, because the risk of fracture of the abutment restorations fabricated by tooth-colored CAD/ CAM materials is unknown, the suitability of applying ceramic restorations as an abutment of RPDs has not yet been clarified. Nevertheless, lithium disilicate glass-ceramic ${ }^{2)}$ and zirconia-based ${ }^{3,4)}$ ceramic and resin composite $^{5)}$ materials have increasingly been used as abutment crowns to support RPDs (Fig. 1). A fouryear retrospective study of veneered zirconia single crowns reported $3 \%$ occlusal rest seat fractures and $11 \%$ veneering porcelain fractures in non-metallic RPD abutment crowns ${ }^{4}$. Moreover, $6.7 \%$ crown fracture and $33 \%$ crown detachment were reported in a three-year retrospective study of monolithic resin composite crowns $\mathrm{s}^{5}$; this study emphasized that the risk of complications was significantly higher for RPD abutment crowns than for RPD non-abutment crowns. Therefore, strict scientific standards have to be established for tooth-colored abutment crowns.

Color figures can be viewed in the online issue, which is available at J-STAGE.

Received Aug 6, 2021: Accepted Dec 16, 2021

doi:10.4012/dmj.2021-224 JOI JST.JSTAGE/dmj/2021-224
A rest seat is prepared on the abutment tooth of RPDs and is loaded through the rest, which might differ from the occlusal contact. The rest seat fracture is affected by the shape of the rest seat. It was reported that the small radius of curvature of the rest surface reduced the fracture resistance of the rest seat ${ }^{6}$. Moreover, the rest seat fracture should be affected by the contact conditions between the rest and rest seat, i.e., the conformity with the rest. This is because if the rest mismatched, the contact locations and areas, and consequently the stress distribution of the rest seat, may be different than expected. The rest and rest seat are not always considered to be accurately fitted in clinical situations because only $24 \%$ of the rest is in contact with the bottom of the rest seat ${ }^{7}$. However, the effect of the contact conditions between the rest and rest seat on the risk of fracture of the ceramic restorative materials has not been clarified.

The fracture mechanism of ceramic restorations is closely related to the first principal stress distribution and its maximum value ${ }^{8)}$. Because the rest seat has an inner concave shape, if the rest contacts both sidewalls prematurely due to mismatch and performs a wedge action, it could cause high tensile stress concentration on the surface of the rest seat, which can induce fractures ${ }^{9}$. Furthermore, when the rest contacts the bottom surface of the rest seat strongly, high compressive stress concentration and localized high tensile stress concentration would be generated at the contact area and induce Hertzian crack ${ }^{10)}$. Therefore, the fracture of restorative materials with a rest seat as the abutment of RPDs might correspond with the fracture resistance of 


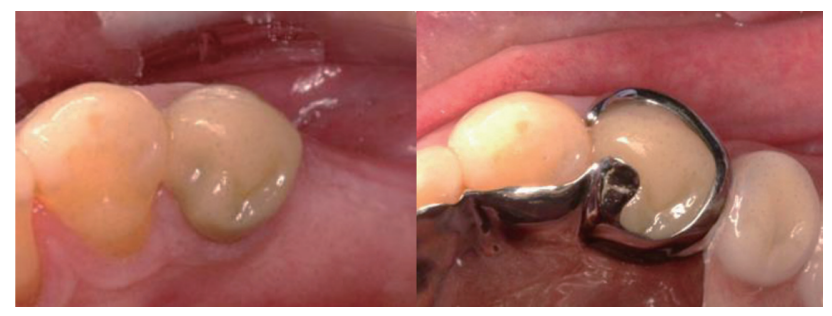

Fig. 1 Photographs of an abutment tooth of the RPD with a rest seat and the RPD.

A first premolar was restored using an all-ceramic surveyed crown with a mesio-occlusal rest seat (left). The clasp-retained RPD with a metal rest was inserted (right).

the restorative materials and the localized contact points, so-called "mismatch contact." However, the relationship between the actual fracture load and calculated stress distribution remains to be clarified.

In this study, we evaluated the fracture risk introduced by preparing the rest seat on CAD/CAM restorative materials as the abutment of RPDs. Using an experimental block that simplifies the configuration of the restorative materials and rest seat, we calculated the stress distribution using the finite element method (FEM). We analyzed the fracture load obtained from the fracture tests by controlling the contact condition between the rest and rest seat. The null hypothesis of this study was that the restorative materials with rest seats in mismatched RPD rests do not influence the fracture resistance or stress distribution in the rest seats made from $\mathrm{CAD} / \mathrm{CAM}$ tooth-colored materials.

\section{MATERIALS AND METHODS}

\section{Fracture test}

The experimental blocks were prepared with four CAD/ CAM restorative materials: feldspathic porcelain (FP; VITABLOCS Mark II, VITA Zahnfabrik, Bad Säckingen, Germany); lithium disilicate glass-ceramic (LD; IPS e.max CAD, Ivoclar Vivadent, Schaan, Liechtenstein); zirconia-based ceramic (ZC; inCoris TZI, Sirona Dental Systems, Bensheim, Germany); hybrid resin composite (HC; Cerasmart 270, GC, Tokyo, Japan). Rest seats with different widths $(2.5,2.6$, and $2.7 \mathrm{~mm})$ were prepared against the metal indenter representing the rest (2.6$\mathrm{mm}$ width) on each block ( $n=14$ for each rest seat width). As a result, the contact conditions between the rest and rest were divided into the accurate fit $(2.6 \mathrm{~mm})$ and mismatch contact of bottom contact $(2.7 \mathrm{~mm})$ and sidewall contact (2.5 mm) (Fig. 2).

Each CAD/CAM materials was cut and shaped into rectangular blocks $(7.0 \times 7.0 \times 3.0 \mathrm{~mm})$ simulating the average occlusal table of a first premolar using a precision cutter (IsoMet ${ }^{\mathrm{TM}}$ Low Speed, Buehler, Lake Bluff, IL, USA) and diamond disk (Cameo disk platinum type 3 and 4, Kemet Japan, Chiba, Japan). Round-shaped occlusal rest seats with three widths $(2.5,2.6$, and $2.7 \mathrm{~mm}), 1.5$ -
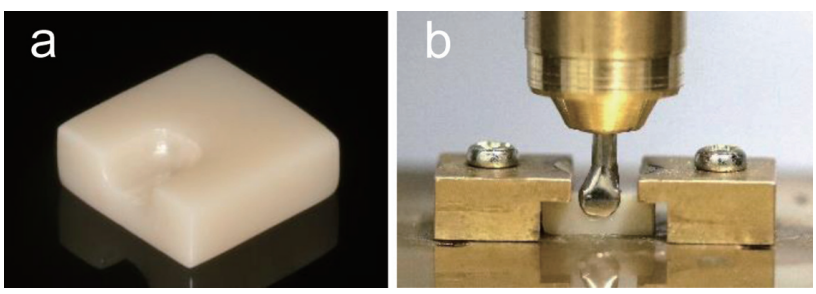

Fig. 2 Photographs of experimental block and fracture test equipment.

(a) Experimental block with a rest seat. (b) The metal indenter was positioned on the rest seat of the block with the special jig, and the vertical load was applied.

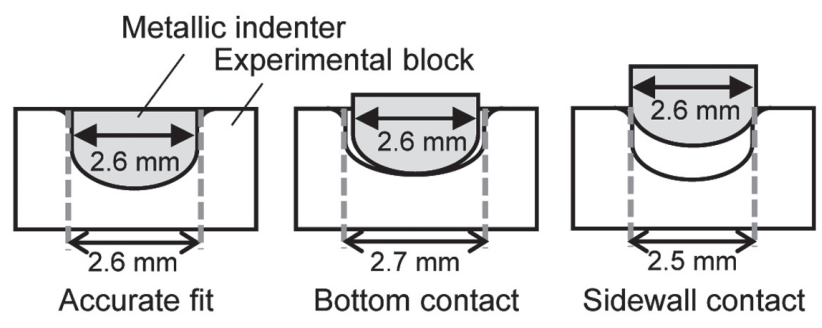

Fig. 3 Schematics of contact conditions.

The rest seat was prepared on the block against the metallic indenter assuming the occlusal rest was in one of three contact conditions: accurate fit (indenter accurately fitted to the rest seat); bottom contact (indenter contacted the bottom of rest seat); sidewall contact (indenter contacted both sidewalls of rest seat).

mm depth, and 3-mm length (Fig. 3) were prepared on the block using a round-headed diamond bur $(\Phi 2.5 \mathrm{~mm}$, D3225, Nihon Seimitsu Kikai Kosaku, Hyogo, Japan) in a milling machine (LittleMilling9, Toyo Associates, Tokyo, Japan). After removing the sharp edges, the plane surfaces, excluding the bottom of the specimens, were polished using a polishing disk (Sof-Lex, Contouring and Polishing Disks, 3M Japan, Tokyo, Japan), and the rest seats were polished using a diamond bur (SF107RD, SHOFU, Kyoto, Japan) and point (StarGloss CA Medium, MORITA, Osaka, Japan). FP, LD, and ZC were polished with a polishing paste (ZIRKOPOL, Pentron Japan, Tokyo, Japan). Before polishing, LD and ZC were fired in a furnace (VACUMAT 40T and ZYRCOMAT, VITA Zahnfabrik) following the manufacturer's parameters. The metal indenters representing the occlusal rest were waxed up on each 2.6-mm-wide rest seat specimen, and finally, they were cast using a silver-palladium-coppergold alloy (Ag-Pd-Cu-Au) (Castwell M.C.12, GC).

The experimental block and metallic indenter were fixed in the custom-made holding unit with vertical orientation, and the holding unit was positioned in a universal testing machine (Autograph AG-X plus, Shimadzu, Kyoto, Japan). Prior to the test, the contact condition between the rest and rest seat was confirmed 
using a silicone impression material (BLUE SILICONE, GC). One operator evaluated all the samples, and those with inferior quality were rejected. Then, the metal indenter was placed on the rest seat, and the vertical load was applied to the block via the holding unit at a crosshead speed of $0.5 \mathrm{~mm} / \mathrm{min}$ until fracture. Videos of the fracture tests were also recorded with a digital camera (EOS Kiss X8i, Canon, Tokyo, Japan). The specimen was considered to fracture when the load decreased by $5 \%$. This timing also coincided with the time when a visible crack appeared and the occurrence of a cracking sound. Finally, representative specimens were sputtered with platinum and palladium and were examined using an optical stereomicroscope (VHX-1000, Keyence, Osaka, Japan) with $\times 100$ magnification to determine the fracture pattern.

Fracture load values were analyzed statistically using SPSS 11.5J for Windows (SPSS, Chicago, IL, USA). We compared the different contact types within the same material using the Kruskal-Wallis test and pairwise comparisons $(\alpha=0.05)$.
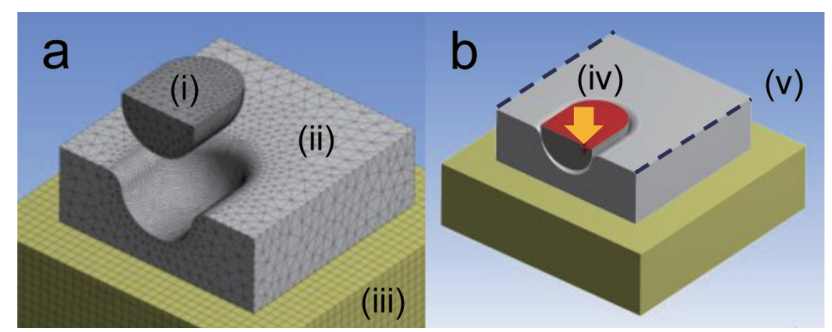

Fig. 4 (a) Finite element models and (b) boundary conditions.

(i) Meshed models of metallic indenter, (ii) experimental block, and (iii) brass stage. The bottom surface of the brass stage was restrained to zero in all directions, and the upper surface and rest seat specimen were in contact without friction. (iv) The rest seat was vertically loaded via a metal rest indenter with frictional contact. (v) The upper side of the rest seat specimen (dotted lines) were vertically restrained to zero.

\section{Finite element analysis}

Three-dimensional finite element models of the experimental block with the rest seat and metallic indenter were constructed to simulate the fracture test (Fig. 4). Each model comprised approximately 36,000 tetrahedral elements, as determined by preliminary convergence tests (Ansys 19.0, ANSYS, Canonsburg, PA, USA). All materials were homogeneous, linearly elastic, and isotropic. The elastic modulus and Poisson's ratio of each material listed in Table $1^{11-15)}$ were entered in the simulation program. The bottom of the brass stage was restrained to zero displacements in all directions, and both sides of the upper surface of the block were restrained to zero in the vertical direction. The contact surface at the interface between the rest and rest seat was assigned a friction coefficient of $0.1^{16-18)}$, and that between the bottom surface of the block and brass stage was assigned a frictionless contact. Vertical loads from 50 to $1,800 \mathrm{~N}$ were applied on the top surface of the metal indenter with restricted horizontal movement, thereby simulating the fracture tests. The first principal stress distribution was calculated using nonlinear contact analyses.

\section{RESULTS}

\section{Fracture test}

Figure 5 showed photographs of the fractured ZC blocks. Fracture modes were classified into a crushing pattern and a split fracture pattern. In the crushing pattern,
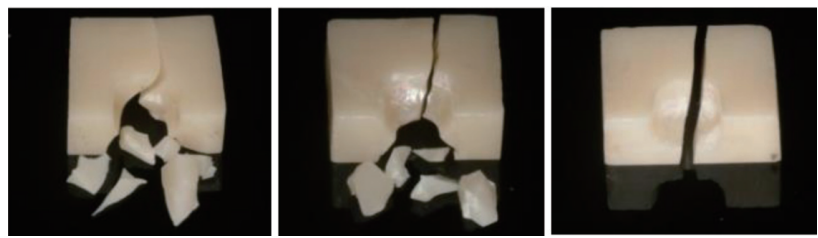

Fig. 5 Photographs of the fractured zirconia block in three contact conditions.

The blocks of the accurate fit and the bottom contact showed the crushing fracture, and the sidewall contact showed a split fracture.

Table 1 Properties of the materials used in this study Brass: Acquired from material library

\begin{tabular}{|c|c|c|c|c|c|}
\hline Material & Abbreviation & $\begin{array}{c}\text { Flexural } \\
\text { strength }(\mathrm{MPa})\end{array}$ & $\begin{array}{c}\text { Young's } \\
\text { modulus (GPa) }\end{array}$ & $\begin{array}{l}\text { Poisson's } \\
\text { ratio }\end{array}$ & Reference \\
\hline Feldspathic porcelain & FP & 170 & 72 & 0.23 & $12,15)$ \\
\hline Hybrid resin composite & $\mathrm{HC}$ & 205 & 9.25 & 0.3 & 14) \\
\hline Lithium disilicate glass ceramic & $\mathrm{LD}$ & 376 & 102 & 0.21 & $12,13)$ \\
\hline Zirconia & $\mathrm{ZC}$ & 1,050 & 216 & 0.3 & 12) \\
\hline $\mathrm{Ag}-\mathrm{Pd}-\mathrm{Au}-\mathrm{Cu}$ alloy & - & - & 89 & 0.3 & 11) \\
\hline Brass & - & - & 110 & 0.34 & - \\
\hline
\end{tabular}


the lower part of the rest seat was crushed into small pieces and it was spread radially from the center of the rest seat surface toward the bottom of the specimen. In the split fracture pattern, the specimen was split in two at the midline. The blocks of the accurate fit and bottom contact fractured with a crushing pattern, and the majority of the sidewall contacts were fractured with a split fracture pattern. These fracture modes were observed in all materials, whereas some composite resins showed many cracks but no crushing.

Figure 6 shows the results of the average fracture loads of each material in contact conditions. Among the materials tested, ZC showed the highest fracture load in all contact conditions. With ZC, the accurate fit revealed the highest fracture load of $(1,412 \pm 365 \mathrm{~N})$, followed by the bottom contact $(1,108 \pm 491 \mathrm{~N})$ and sidewall contact $(880 \pm 432 \mathrm{~N})$. There was a significant difference between the accurate fit and sidewall contact $(p<0.05)$. The contact conditions for each material were same.

Figure 7 shows representative digital microscope images of the fracture surfaces around the rest seats. Fracture origins (Fig. 7, asterisks) below the bottom

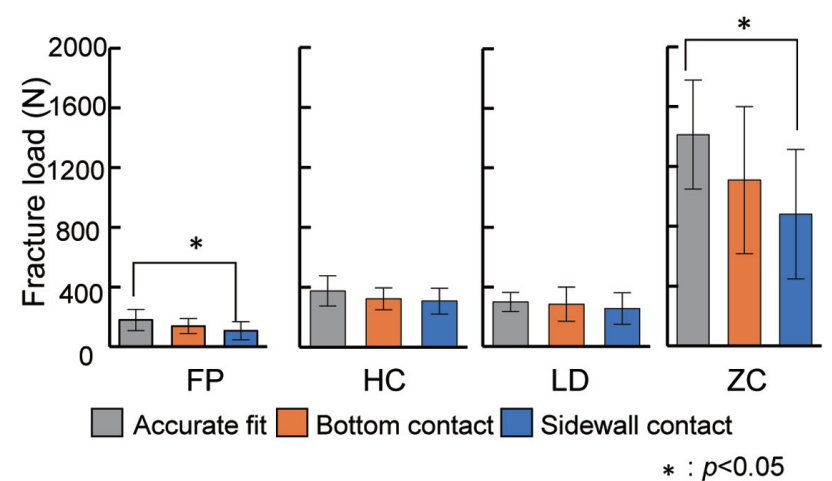

Fig. 6 Results of fracture load of experimental block with rest seat in the three contact conditions for each material.

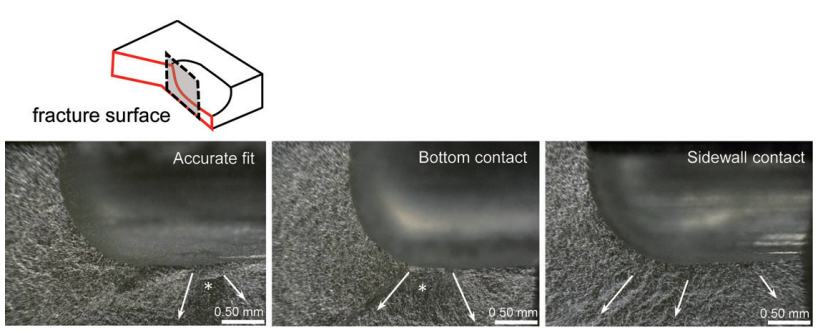

Fig. 7 Illustration of the observation part and the digital microscopic images of the fracture surface of the experimental block.

The digital microscopic images show the fractured surfaces of the feldspathic porcelain coated with platinum and palladium in each contact condition. Asterisks indicate crack initiation points. White arrows indicate hackle patterns that follow the direction of crack propagation. of the rest seat and crack propagation (Fig. 7, arrows) radiating downward from the crack origin were observed in the accurate fit and bottom contact. Obvious fracture origins could not be detected in the sidewall contact; however, crack propagation from the rest seat surface to the bottom surface of the specimen was observed. These tendencies were observed in ZC and LD. Meanwhile, some of the fracture surfaces of $\mathrm{HC}$ showed multiple crack origins in one fracture surface, regardless of the contact condition. The first crack origin could not be identified, but the cracks propagated from the surface of the rest seat to the bottom surface.

\section{Finite element analysis}

Figure 8 shows the distribution of the first principal stress of the ZC block when a load of $100 \mathrm{~N}$ was applied to the metal indenter. In the bottom contact (Fig. 8, center), the high compressive stress was concentrated in the center of the bottom surface of the rest seat, in which the contact with the rest and localized high tensile stress concentrated around the contact area. In the sidewall contact (Fig. 8, right), high tensile stress was concentrated not only locally around the contact area on the sidewalls but also at the bottom center of the rest seat away from the contact area in the sidewall contact (Fig. 8, right). In the accurate fit, the maximum tensile stress was found at the bottom surface of the rest seat. However, the stress range was smaller throughout the rest seat than in the other conditions (Fig. 8, left). The stress concentration tendency was the same in each contact condition regardless of material.

Relationship between the maximum value of the first principal stress at the bottom of the rest seat and fracture load

The maximum stress value of the bottom of the rest seat was localized near the center in all groups. Figure 9 shows a plot of the applied load as a function of the maximum value of the first principal stress generated in the center of the bottom surface of the rest seat for each material. The curve of sidewall contact (blue line) was
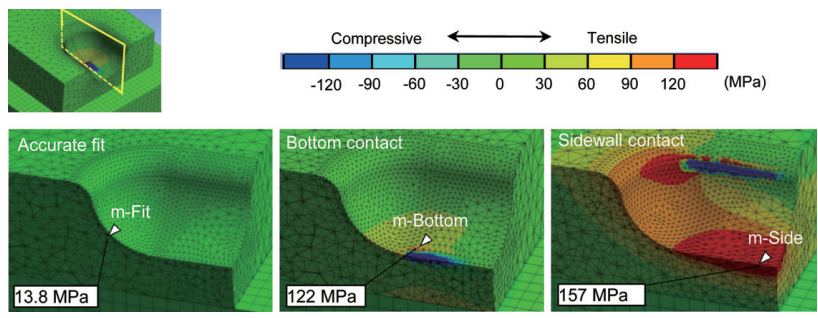

Fig. 8 First principal stress distribution of the zirconia in three contact conditions under a $100-\mathrm{N}$ load.

The cross-sectional view indicated by the yellow square in the upper figure was shown in the lower figures. The white arrows labeled m-Fit, m-Bottom, and $\mathrm{m}$-Side indicate the point that shows the maximum value of the first principal stress at the bottom of the rest seat. 

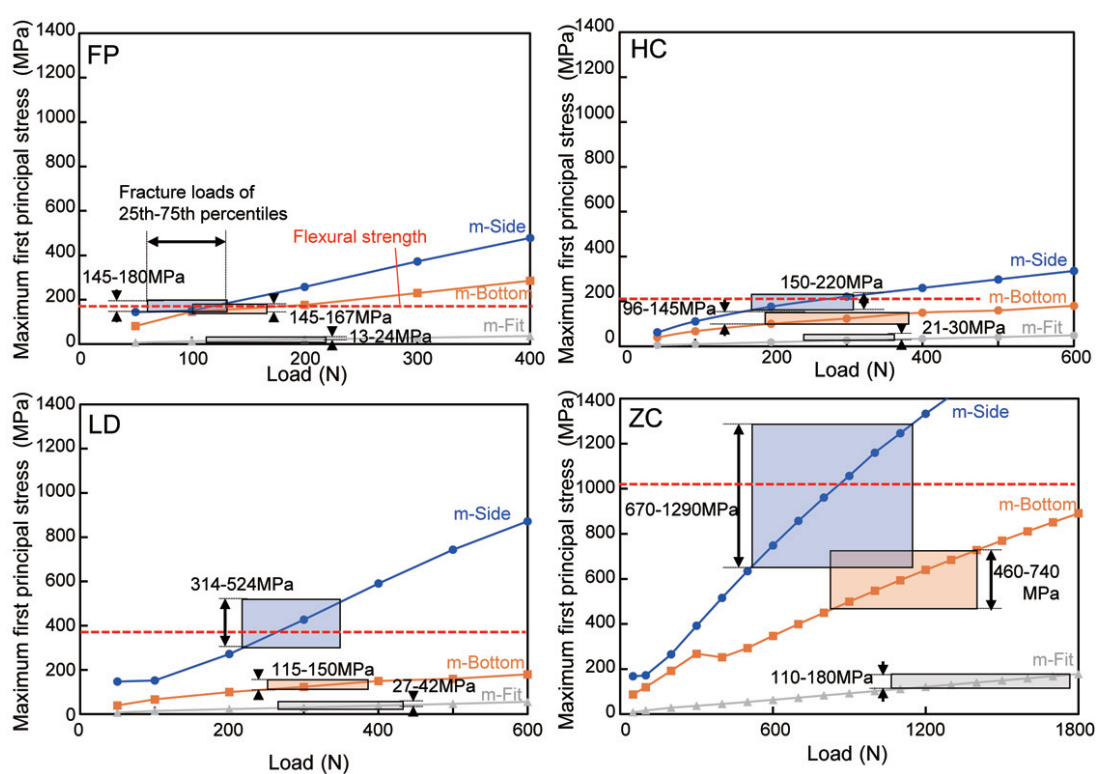

Fig. 9 Plots of maximum first principal stress at the points labeled m-Fit, m-Bottom, and m-Side in Fig. 8 versus applied load at the bottom of the rest seat.

In the areas surrounded by the square, the width is the range of the fracture loads of 25 th to 75 th percentiles obtained from the fracture tests in each contact condition, and the height is the maximum first principal stress range corresponding to that load range. The horizontal dotted line indicates the flexural strength of each material.

the highest, and the accurate fit curve (gray line) was the lowest. The width of the square indicates the fracture load range of the 25 th to 75 th percentile obtained in the fracture test. The height is the first principal stress range calculated by FEM equivalent to the width of the fracture load range. In each material, the first principal stress range corresponding to the fracture load depended on the contact condition; the highest and lowest value was obtained for the sidewall contact and accurate fit. As a reference, the flexural strength obtained from the previous reports is shown (horizontal dotted line). Moreover, at the sidewall contact of all materials, the first principal stress range corresponding to the fracture load included the flexural strength line of each material. In contrast, those of the bottom surface and accurate fit were lower than the flexural strength.

\section{DISCUSSION}

The fracture resistance of the restorative materials with rest seats was clearly affected by the contact conditions with the rest. Especially with the sidewall contact, the fracture resistance was significantly decreased. The fracture behaviors of the rest seat in each contact condition depended on the stress distribution. Therefore, the null hypothesis of this study was rejected.
Relationship between fracture load and first principal stress on the bottom of the rest seat

In addition to the in vitro fracture test, we performed a 3D finite element analysis to investigate the fracture mechanism of the block with the rest seat in the contact condition. If the fracture is caused by tensile stress of the same mechanism, the fracture should occur at the exact first principal stress. Unfortunately, tensile strength of some brittle materials can not be easily measured. Therefore, the flexural strength is often measured for such materials instead of tensile strength. Since the flexural strength was calculated by the flexural moment at the tensile side in the flexural test, flexural strength is strongly related to tensile stress ${ }^{19,20)}$. Moreover, flexural strength is the most commonly measured mechanical property of brittle materials. Consequently, in this study, we focused on the first principal stress and interpreted the fracture mechanism using the flexural strength (Table 1) as a reference value ${ }^{21)}$. As shown in Fig. 9, the areas surrounded by the square are the range of the width of the fracture loads of 25 th to 75 th percentiles obtained from the fracture tests in each contact condition, and the height of the maximum first principal stress range corresponding to that load range. The horizontal dotted line indicating the flexural strength intersected this blue square area of m-Side in all materials. This result suggested that the fracture at the sidewall contact strongly relates to the flexural strength of each material. 
The sidewall contact showed a unique vertical fracture from the bottom of the rest seat in which the block split in half (Figs. 5 and 7, right). Furthermore, the tensile stress leading to the fracture was generated at the bottom of the rest seat, far from the contact area in the sidewall contact (Fig. 8, right). The reason was that the maximum tensile stress at the center of the bottom surface increased owing to the wedging effect due to the loads on both sidewalls of the rest seat in a V-shape, potentially leading to a vertical fracture ${ }^{22}$. In this way, because the fracture originated from the predominant tensile stress due to the deformation of the bottom of the rest seat, the theoretical first principal stress range corresponding to the actual fracture load was reflected the flexural strength at the sidewall contact.

Based on the fractography of the bottom contact (Figs. 5 and 7, center), the fact that the Hertzian contact produces a cone $\operatorname{crack}^{23-25)}$ was considered to have caused the rest seat fracture, and the bottom of the rest seat under the contact area was crushed and fractured. In the stress analysis, the localized tensile stress concentration due to the compressive stress of the contact area (Fig. 8, center) is almost the same as the crack initiation part of the fractography. Therefore, it was considered that this tensile stress generated a crack and propagated to the bottom as the crack progressed. Moreover, it has been reported that the reproduction of Hertzian contact fracture requires a finite element analysis ${ }^{10)}$. Furthermore, fractures caused by compression, the compressive and shear stresses must be further analyzed.

The stress analysis results of the accurate fit conditions showed minimal stress changes (Fig. 8, left). This result is in good agreement with the results of the experimental fracture test, which indicated that the fracture resistance was the highest in all contact conditions (Fig. 6). Nevertheless, the difference in fracture load was not as significant as the difference in stress distribution. Although we confirmed the fitting accuracy before the tests, it was difficult to obtain a good fit in the experimental fracture test, suggesting that partial mismatches, such as that in sidewall contact and bottom contact, occurred in a small area.

\section{Risk assessment of each material}

In this study, the restorative materials with accurately fitted rest had the highest fracture resistance, which prevents stress concentration. Moreover, when the rest contacted both sidewalls of the rest seat, the highest stress concentration occurred at the bottom of the rest seat, resulting in the lowest fracture resistance. Therefore, it was suggested that contacts at both sidewalls had a high risk of fracture. In clinical practice, various contacts can occur between the rest and the rest seat. In this study, we focused on two simple fracture patterns depending on the contact condition: fracture between two contact points and fracture around the contact point. However, other contact conditions could also be explained by the fracture mechanism adopted in this study. It is crucial to examine the contact between the rest and rest seat in clinical practice. To reduce stress concentration and prevent fracture, it is necessary to prevent contact with the sidewall or to increase the contact area.

Furthermore, the maximum value of the first principal stress at the bottom of the rest seat when the actual fracture load was applied at the sidewall reflected the flexural strength of the material. Therefore, when evaluating the fracture risk of a monolithic ceramic crown with a rest seat, it may be possible to estimate the relative fracture resistance of comparative materials from flexural strength and the first principal stress at the bottom of the rest seat, assuming the sidewall contact $^{26,27)}$. However, the relationship between the fracture resistance value suggested by flexural strength and the occlusal force must be verified experimentally.

The mean occlusal force for the patient treated with RPDs was $173.6 \mathrm{~N}^{28)}$, while that for healthy and young adults in the posterior region, it was around $700 \mathrm{~N}^{29}$. Therefore, from the results of the present study, resin composite, lithium disilicate, and zirconia ceramics might withstand the average occlusal force in patients with RPDs assuming the average occlusal force of patients with RPDs. Furthermore, zirconia, which has resistance to the highly occlusal force of healthy dentition, should be the safest material.

However, the load applied to the rest seat from the rest that propagated the occlusal force applied to the denture teeth is unknown. Moreover, numerous factors contribute to the fracture of monolithic crowns, including the rest seat design, occlusal thickness of ceramics, abutment preparation design, the elastic modulus of the abutment tooth ${ }^{30-32)}$, rest materials, adhesion of cement, and the presence of defects. Furthermore, the coefficient of friction between the rest and the rest seat is affected by the surface properties. In the case of sidewall contact, the preliminary analysis demonstrated a significant decrease in the first principal stress at the bottom of the rest seat with the increasing coefficient of friction. Therefore, we assumed that the rest seat was smooth and used 0.1 as the coefficient. In this study, we focused on the difference in stress distribution and fracture resistance peculiar to the rest seat under various contact conditions between the rest and the rest seat; therefore, the simply shaped specimens with rest seats were used in the static loading fracture test. Moreover, to better understand the fracture resistance of rest seat materials, thermal cycling, and repeated fatigue tests under wet conditions similar to the in-vivo environment and further research considering various factors is needed.

\section{CONCLUSION}

$\mathrm{CAD} / \mathrm{CAM}$ restorative materials with the mismatch of the rest caused a decrease in fracture resistance. When the rests are in contact with the sidewalls of the rest seat, the fracture resistance is significantly reduced owing to the tensile stress concentration at the bottom of the rest seat. The restorations with the occlusal rest seat made of resin composite, lithium disilicate, and zirconia ceramics can withstand the average occlusal 
force of patients with RPDs. Furthermore, zirconia can withstand the occlusal force of the posterior region of healthy dentition.

\section{ACKNOWLEDGMENTS}

This work was partially supported by JSPS KAKENHI (Grant Number 17K17155 and18K09676).

\section{REFERENCES}

1) Rudd RW, Bange AA, Rudd KD, Montalvo R. Preparing teeth to receive a removable partial denture. J Prosthet Dent 1999; 82: 536-549.

2) Yoon TH, Chang WG. The fabrication of a CAD/CAM ceramic crown to fit an existing partial removable dental prosthesis: A clinical report. J Prosthet Dent 2012; 108: 143-146.

3) Carracho JF, Razzoog ME. Removable partial denture abutments restored with all-ceramic surveyed crowns. Quintessence Int 2006; 37: 283-288.

4) Pihlaja J, Napankangas R, Kuoppala R, Raustia A. Veneered zirconia crowns as abutment teeth for partial removable dental prostheses: a clinical 4-year retrospective study. J Prosthet Dent 2015; 114: 633-636.

5) Miura S, Kasahara S, Yamauchi S, Katsuda Y, Harada A, Aida $\mathrm{J}$, et al. A possible risk of CAD/CAM-produced composite resin premolar crowns on a removable partial denture abutment tooth: A 3-year retrospective cohort study. J Prosthodont Res 2019; 63: 78-84.

6) Manchester JA, Chung KH, Brudvik JS, Ramos V Jr, Chen YW. Fracture resistance of cingulum rest seats in CADCAM tooth-colored crowns for removable partial denture abutments. J Prosthet Dent 2019; 121: 828-835.

7) Dunham D, Brudvik JS, Morris WJ, Plummer KD, Cameron SM. A clinical investigation of the fit of removable partial dental prosthesis clasp assemblies. J Prosthet Dent 2006; 95: 323-326.

8) Heintze SD, Monreal D, Reinhardt M, Eser A, Peschke A, Reinshagen J, et al. Fatigue resistance of all-ceramic fixed partial dentures - Fatigue tests and finite element analysis. Dent Mater 2018; 34: 494-507.

9) Munari LS, Bowles WR, Fok ASL. Relationship between canal enlargement and fracture load of root dentin sections. Dent Mater 2019; 35: 818-824.

10) Chen SY, Farris TN, Chandrasekari S. Contact mechanics of hertzian cone cracking. Int J Solids Structures 1995; 32: 329340.

11) Matsuo S, Watari F, Ohata N. Fabrication of a functionally graded dental composite resin post and core by laser lithography and finite element analysis of its stress relaxation effect on tooth root. Dent Mater J 2001; 20: 257-274.

12) Ren L, Zhang Y. Sliding contact fracture of dental ceramics: Principles and validation. Acta Biomater 2014; 10: 32433253.

13) Belli R, Wendler M, de Ligny D, Cicconi MR, Petschelt A, Peterlik $\mathrm{H}$, et al. Chairside CAD/CAM materials. Part 1: Measurement of elastic constants and microstructural characterization. Dent Mater 2017; 33: 84-98.
14) Lucsanszky IJR, Ruse ND. Fracture toughness, flexural strength, and flexural modulus of new CAD/CAM resin composite blocks. J Prosthodont 2020; 29: 34-41.

15) Al-Thobity AM, Gad MM, Farooq I, Alshahrani AS, AlDulaijan YA. Acid effects on the physical properties of different CAD/CAM ceramic materials: An in vitro analysis. J Prosthodont 2021; 30: 135-141.

16) Sato Y, Abe Y, Yuasa Y, Akagawa Y. Effect of friction coefficient on Akers clasp retention. J Prosthet Dent 1997; 78: 22-27.

17) Dąbrowa T, Dobrowolska A, Wieleba W. The role of friction in the mechanism of retaining the partial removable dentures with double crown system. Acta Bioeng Biomech 2013; 15: 43-48.

18) Ludovichetti FS, Trindade FZ, Werner A, Kleverlaan CJ, Fonseca RG. Wear resistance and abrasiveness of CAD-CAM monolithic materials. J Prosthet Dent 2018; 120: 318.e1-.e8.

19) Ban S, Anusavice KJ. Influence of test method on failure stress of brittle dental materials. J Dent Res 1990; 69: 17911799.

20) Ling L, Ma Y, Malyala R. A novel CAD/CAM resin composite block with high mechanical properties. Dent Mater 2021; 37: 1150-1155.

21) Lan TH, Chen PH, Fok ASL, Chen YF. Contact fracture test of monolithic hybrid ceramics on different substrates for bruxism. Dent Mater 2022; 38: 44-56.

22) Sornsuwan T, Swain MV. Influence of occlusal geometry on ceramic crown fracture; role of cusp angle and fissure radius. J Mech Behav Biomed Mater 2011; 4: 1057-1066.

23) Kelly JR. Clinically relevant approach to failure testing of allceramic restorations. J Prosthet Dent 1999; 81: 652-661.

24) Zhou J, Mah J, Shrotriya P, Mercer C, Soboyejo WO. Contact damage in an yttria stabilized zirconia: Implications. J Mater Sci Mater Med 2007; 18: 71-78.

25) Coldea A, Swain MV, Thiel N. Hertzian contact response and damage tolerance of dental ceramics. J Mech Behav Biomed Mater 2014; 34: 124-133.

26) Ona $M$, Watanabe $C$, Igarashi $Y$, Wakabayashi N. Influence of preparation design on failure risks of ceramic inlays: A finite element analysis. J Adhes Dent 2011; 13: 367-373.

27) Murakami N, Wakabayashi N. Finite element contact analysis as a critical technique in dental biomechanics: A review. J Prosthodont Res 2014; 58: 92-101.

28) Miyaura K, Morita M, Matsuka Y, Yamashita A, Watanabe T. Rehabilitation of biting abilities in patients with different types of dental prostheses. J Oral Rehabil 2000; 27: 10731076.

29) Padma S, Umesh $\mathrm{S}$, Asokan $\mathrm{S}$, Srinivas $T$. Bite force measurement based on fiber Bragg grating sensor. J Biomed Opt 2017; 22: 1-6.

30) Lawn BR, Deng Y, Miranda P, Pajares A, Chai H, Kim DK. Overview: Damage in brittle layer structures from concentrated loads. J Mater Res 2002; 17: 3019-3036.

31) Yucel MT, Yondem I, Aykent F, Eraslan O. Influence of the supporting die structures on the fracture strength of allceramic materials. Clin Oral Investig 2012; 16: 1105-1110.

32) Zhang Y, Lawn BR. Novel zirconia materials in dentistry. J Dent Res 2018; 97: 140-147. 